first paper, practised the double operation on the principle and with the views explained and advocated by myself. Nothing can be more manifest, from the whole tenor and details of his former communications (as may be seen by referring to The LanceT of Sept. 12 to Oct. 10 inclusive), than that he had never been in the habit of dividing the rectus of the apparently straight eye for the complete rectification of the other eye, when it had been imperfectly straightened by the first operation, viz., upon itself, in which the peculiar value of my operation consists ; on the contrary, he has merely been in the habit of dividing the rectus of the second eye, if the second eye uas strabismal, not otherwise. This is merely a repetition of the operation, and undertaken on the same grounds as that on the first eye, and, so far as I know, was first practised in this country by Mr. Lucas, and mentioned by him in The Lancet, three or four months ago. I am, Sir, your obedient servant,

Thomas Elliot, M.R.C.S.L.

23, English.street, Carlisle, Oct. 12, 1840.

\section{ANOTHER MODE OF OPERATING FOR STRABISMUS.}

\section{To the Editor of THE LANCET.}

SIR :-I shall feel obliged by your insert. ing a few remarks on the subject of the division of the recti muscles in cases of strabismus convergens, and on the method adopted in performing this operation by ny friend and instructor, Mr. Lane, of Grosvenor-place.

The operation I now submit for your perusal, appears to me to possess great advantages over any other method now in practice; by it the division of the recti muscles may be effected by one assistant and with one instrument, and without any previous division of the conjunctiva. Ten or twenty seconds is the time usually required to perform this operation. In thirteen cases which occurred in my own practice, three only required a longer period: in these three cases, in addition to the section of the internal rectus, it was necessary to divide a few of the tendinous fibres of the superior and inferior recti muscles; this was effected by passing a director under these muscles, and dividing their inner edges with a pair of sharp-pointed scissors. The plan I adopt in the performance of this operation is as follows:-An assistant, who is placed be. hind the patient, lightly closes the sound eye with one hand, and with the other depresses the lower lid of the organ affected. Supposing the right eye to require the operation, I place myself before, and rather to the right, of my patient; with the fore finger of my left hand I raise and secure the upper eyelid : the patient is now desired to turn the eye outwards, which is generally effected without difficulty; the point of the knife (which is described below) is now fixed upon the conjunctiva, in the interval between the inferior and interual recti muscles. By depressing the hand and cautiously pass. ing the knife onwards, the conjunctiva is made to pass with the knife under the edge of the internal rectus : by using slight force the membrane gives way before the point of the knife, leaving it between the muscle and sclerotic coat; then, by gentle manipulation, the point appears at the interval between the iuternal and superior rectus mus: cle, still covered by the mucous membrane, which is pierced as before; the muscle and membrane now lie upon the edge of the knife, and are divided by moving the instru. ment onwards. The best idea I can give of the instrument referred to is, to compare its form to that of a sickle, with this exception, that it is not quite so much curved, and ter. minates almost imperceptibly in a partiallyblunt point: the pattern may be had of Messrs. Philp and Wicker, of St. James'sstreet.

On perusing The Lancet for the 5 th of Sept., I find the following objections advanced to the use of the knife, without the aid of the director or hook :- " To the use of a knife without a director or hook, the objections are so obvious, that $I$ believe few surgeons have tried the experiment, though, I regret to say, I have seen a surgeon of very great experience attempt this; and the consequences were, as might be expected, a wound in the sclerotica, and the escape of the vitreous humour." Such a catastrophe, with. out doubt, may occur; but I presume the knife used on the unfortunate occasion referred, must have been in shape and make like the sharp-pointed bistoury; but with the knife I have just described such an error cannot occur, unless very undue force be used, and that in quite a wrong direction.

In the operation for lithotomy, were the surgeon ignorant of the manner the knife should be lateralised, the pudic artery would no doubt be divided; so would the sclerotic coat in this operation be punctured, if the operator forgot that the point of his knife was against the sclerotic coat instead of the conjunctiva. The instrument used by Mr. Lane and myself has its point so far blunted, that it cannot be made to enter the dense sclerotic coat, unless very great violence be exerted, at the same time it is sufficiently pointed to pass with facility through the delicate texture of the conjunctiva. I am, Sir, your obedient servant,

Herbert Fagg.

Bayswater, Oct. 15, 1840. 